\title{
Children with chronic-refractory autoimmune cytopenias: a single center experience
}

\author{
Tuba Hilkay Karapınar ${ }^{1 \oplus}$, Ersin Durgun ${ }^{1 \oplus}$, Yeşim Oymak ${ }^{1 \oplus}$, Nesrin Gülez ${ }^{2 \oplus,}$ \\ Yılmaz Ay ${ }^{1 \oplus}$, Ferah Genel ${ }^{2 \oplus}$, Salih Gözmen ${ }^{1 \oplus}$, Erkin Serdaroğlu ${ }^{3 \oplus,}$ \\ Sultan Aydın Köker ${ }^{1 \odot}$, Ersin Töret ${ }^{1 \oplus}$, Canan Vergin ${ }^{1 \odot}$ \\ Departments of ${ }^{1}$ Pediatric Hematology-Oncology, ${ }^{2}$ Pediatric Immunology and ${ }^{3}$ Pediatric Nephrology, Dr. Behçet Uz Children Research \\ and Training Hospital, Izmir, Turkey.
}

\begin{abstract}
Background and objectives. Autoimmune cytopenias are a group of heterogeneous disorders characterized by immune-mediated destruction of one or more hematopoietic lineage cells. The differential diagnosis of children with autoimmune cytopenias requires much time and laboratory investigations. The aim of the present study was to evaluate the clinical course and significance of autoimmune cytopenias due to immunodeficiency or autoimmune diseases in children at a single children's hospital.
\end{abstract}

Method. Between February 1997 and September 2015, chronic/refractory autoimmune cytopenias patient data were evaluated retrospectively. Twenty-three patients were assessed in this study.

Results. The median duration of following was 2.6 years (4 months-18.5 years). The median age of diagnosis was 3.1 years (6 months-16 years). A total of 13 patients (56.5\%) had single-lineage and $10(46.5 \%)$ had multilineage cytopenias. The most frequent single-lineage cytopenia was thrombocytopenia, followed by anemia. In 22 of the patients, cytopenias was detected before the primary diseases. All of the patients were treated with corticosteroids or intravenous immune globulin as first-line treatment. Ten patients (43.5\%) needed second or further-line immunosuppressive therapies that patients diagnosed as systemic lupus erythematosus, hypogammaglobulinemia, or common variable immunodeficiency. A total of 8 patients (34.7\%) recovered from autoimmune cytopenias after the treatment of primer disease. Cytopenias were continued in 14 patients.

Conclusion. Cytopenia may be the first finding of an immunodeficiency or autoimmune disease and primary disease may be diagnosed in the clinical course. Taking the new targeted treatment options into consideration; early diagnosis is likely to become more important in the near-future in order to begin the treatment for the underlying disease as early as possible.

Key words: immune cytopenia, childhood, immune deficiency, autoimmunity.

Autoimmune cytopenias are a group of heterogeneous disorders characterized by immune-mediated destruction of one or more hematopoietic lineage cells. In some instances, they are idiopathic or occur as a manifestation of other underlying disorders, such as autoimmune diseases, immunodeficiency, autoimmune lymphoproliferative syndrome

$凶$ Tuba Hilkay Karapınar

thkarapinar@yahoo.com

Received 19th March 2019, revised 7th June 2019, accepted 5th July 2019.
(ALPS), tumors, medications (penicillin, cephalosporins, sulphonamides, nonsteroidal anti-inflammatory drugs, etc) or infections. ${ }^{1-3}$ Most children with autoimmune cytopenias have idiopathic disease. However, sometimes, the hematological manifestations precede the clinical onset of underlying immune disorders. So, the differential diagnosis of children with autoimmune cytopenias requires much time and laboratory investigations. ${ }^{4-6}$

The management of patients with chronicrefractory autoimmune cytopenias is 
heterogeneous and complex. Typically, these patients are treated with nonspecific immune suppression. Some of those patients respond to first-line immunosuppressive therapy, although many of them require second or further-line therapies. The primary goal of the therapies is directed at the underlying cause of the autoimmunity. ${ }^{4,7}$ However, therapies of autoimmune cytopenias may be insufficient still more, because auto-immunity has been increasingly recognized as an important component of immune disorders., ${ }^{2,48}$

There have been a few reports of chronic/ refractory autoimmune cytopenias in childhood. ${ }^{2,49-11}$ Therefore, the aim of this study was to evaluate the clinical course and significance of autoimmune cytopenias due to immunodeficiency or autoimmune diseases in children followed up at our hospital.

\section{Material and Methods}

\section{Study design, selection of patients and definitions}

A total of 337 files of information belonging to patients with chronic or refractory autoimmune cytopenias except tumors were evaluated retrospectively at our hematology department between February 1997 and September 2015. Cytopenias due to medications, infections or idiopathic were excluded. Ultimately, patients with immune deficiency or autoimmune diseases (23 patients) were included in this study.

Cytopenias were the reduction of the blood cell typesin the peripheralblood. Thrombocytopenia was defined as a platelet count $<100 \times 10^{9} / \mathrm{L}$ according to an International Working Group criteria. $^{12}$ Autoimmune hemolytic anemia (AIHA) was defined as anemia (hemoglobin $<10$ $\mathrm{g} / \mathrm{dl}$ ) with evidence of hemolysis (reticulocytosis and positive direct antiglobulin test). The lower limit of the absolute number of neutrophils was accepted as $1000 / \mu \mathrm{L}$ in infants and $1500 / \mu \mathrm{l}$ in children over 12 months of age. ${ }^{13}$ The diagnosis of autoimmune neutropenia (AIN) was accepted a diagnosis of exclusion. When the cytopenias had persisted more than 3 months, they were accepted as chronic or refractory cytopenias. ${ }^{12-14}$

The diagnosis of Common variable immunodeficiency (CVID) was based on 4 criteria: Age above 4 years, low serum IgG (Immunoglobulin G; reduced by more than two standard deviations below the mean for age), with or without low serum $\operatorname{IgA}$ (Immunoglobulin A) diminished antibody response to immunizations, and exclusion of other potential immune diseases. ${ }^{15}$ Combined immune deficiency was accepted as evidence of a profound $\mathrm{T}$ cell defect along with evidence of a humoral defect. ${ }^{16}$ Patients fulfilling the revised diagnostic criteria for ALPS were classified as ALPS patients: Chronic non-malignant lymphoproliferation, elevated double negative $\mathrm{T}$ cells (CD4-/CD8-, CD3+, TCR $\alpha \beta+$ ) in peripheral blood circulation and defective in vitro Fas-mediated associated. ${ }^{17}$ Systemic lupus erythematosus (SLE) was diagnosed according to Systemic Lupus International Collaborating Clinics classification criteria. ${ }^{18}$

\section{Statistical Methods and Ethical Permission}

Data were analyzed using SPSS 15.0. The results are presented as the mean, standard deviation, median, absolute number, or percentile. Comparison of median values between independent groups was carried out with the Mann Whitney test. This study was approved by local ethics committee of the Dr. Behçet Uz Children's Research and Training Hospital (number: 2018/233).

\section{Results}

Our protocol included evaluation of the following variables for chronic- refractory cytopenias: Children were evaluated using a standard diagnostic approach, obtained thorough history, physical examination, laboratory screening tests (completeblood count, direct antiglobulin test, liver and renal function tests, measurements of immunoglobulin (IgA, IgM, IgG, and IgE), C3 and C4 complements 
and antinuclear antibody levels, standard peripheral blood flow cytometry analysis $\left(\mathrm{CD}^{+}\right.$, $\mathrm{CD}^{+}, \mathrm{CD}^{+}, \mathrm{CD}^{+} 9^{+}, \mathrm{CD} 16^{+} 56^{+}$and if necessary double negative $\mathrm{T}$ cells), serology of several viral pathogens (TORCH, EBV, hepatitis, and HIV) and bone marrow aspiration evaluation (morphologic, cytogenetic).

The patients with immune thrombocytopenia (ITP) were treated with corticosteroids $(30 \mathrm{mg} /$ $\mathrm{kg} / \mathrm{d}$, 3 days, followed 3 days $20 \mathrm{mg} / \mathrm{kg} / \mathrm{d}$, then 2 days $10 \mathrm{mg} / \mathrm{kg} / \mathrm{d}$, pulse methylprednisolone) or a single dose of intravenous immune globulin (IVIG) at $1 \mathrm{~g} / \mathrm{kg}$ as first-line treatment. Further use of IVIG was dependent on clinical response to the initial dose. The patients with AIHA were treated much like ITP as first-line treatment. The treatment of AIN was oral or intravenous antibiotics depending on the severity of bacterial infections as first line treatment. For refractory cases of ITP, AIHA, or both, second or furtherline immunosuppressive therapies including rituximab, mycophenolate mofetil (MMF), cyclosporine $\mathrm{A}$, azathioprine, and chloroquine have been used.

All of the patients were followed up for a maximum 18.5 years or until they recovered from cytopenia. The median duration of following was 32 months (between 4 months-18.5 years). Twenty-three of the patients with chronic or refractory autoimmune cytopenias (6.8\%) had an immune deficiency or an autoimmune disease. All of the patients were younger than 18 years of age at initial application. The

Table I. Cytopenia detected peripheral blood cell lines.

\begin{tabular}{lc}
\hline Cell lines & $\begin{array}{c}\text { Number } \\
\text { of patients } \\
(\mathrm{n}=23)\end{array}$ \\
\hline Platelets & 9 \\
Neutrophils & 1 \\
Erythrocytes & 3 \\
Erythrocytes + Neutrophils & 2 \\
Erythrocytes + Platelets & 5 \\
Platelets+ Neutrophils & 2 \\
Erythrocytes + Neutrophils+ Platelets & 1 \\
\hline
\end{tabular}

median age of diagnosis was 3.1 years (between 6 months- 16 years) and the ratio of male/female was 1.3. A total of 13 patients (56.5\%) had singlelineage cytopenias and 10 patients (46.5\%) had multi-lineage cytopenias (Table I).

A total of 22 patients had cytopenia at initial application. During follow-up, it took 0-77 months to identify the underlying diseases beneath the cytopenias. Two of them were diagnosed with SLE at initial application. So, excluding these 2 patients, primary diseases were diagnosed a median 5 months after cytopenias had occurred. Only one patient was initially diagnosed as CVID, in this case cytopenia developed during the following years.

Last diagnoses of the patients are given in Table II. Two of the patients were diagnosed as ALPS, 3 of the patients were diagnosed as CVID, 8 of the patients were diagnosed as hypogammaglobulinemia, 5 of the patients were diagnosed as SLE, 2 of the patients were diagnosed as Wiskott-Aldrich syndrome (WAS), one was diagnosed as combined immunodeficiency, one was diagnosed as DiGeorge syndrome, and one was diagnosed as celiac disease. DiGeorge syndrome and Wiskott-Aldrich syndrome were diagnosed by genetic mutation analysis.

All of the patients were treated with corticosteroids or IVIG as first-line treatment. Ten patients $(43.5 \%)$ needed second or further-line immunosuppressive therapies. These patients were diagnosed as SLE, hypogammaglobulinemia, or CVID. Rituximab was used on 6, MMF was used on 5, cyclosporine A was used on 3, and azathioprine was used on 3 patients. Two patients had undergone splenectomy. Only one patient with neutropenia needed granulocyte-colony stimulating factor treatment. He was diagnosed with combine immune deficiency (Table II).

A total of 8 patients $(34.7 \%)$ recovered from autoimmune cytopenias after the treatment of primer disease. Of these 8 


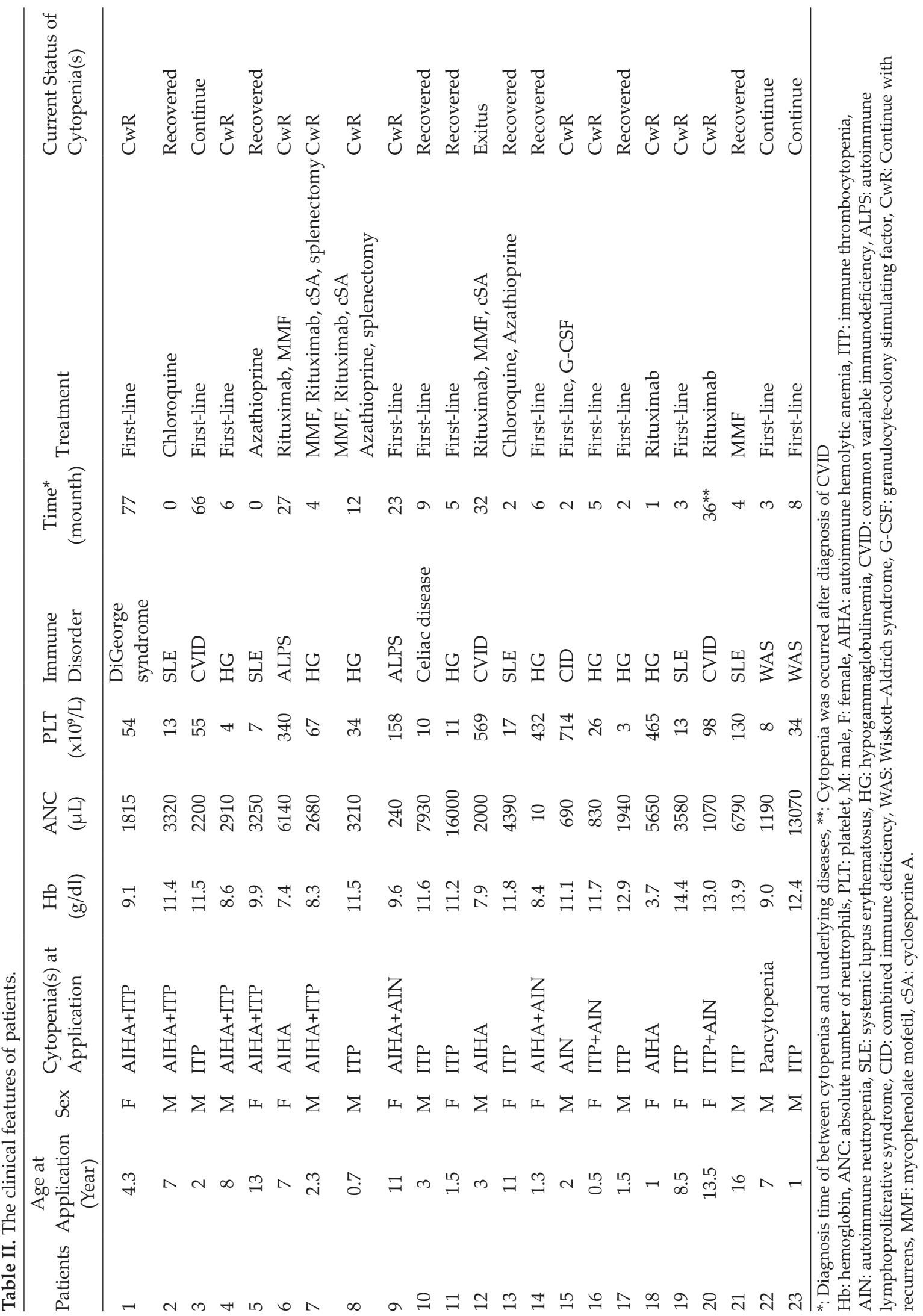


patients; 4 were diagnosed with SLE, 3 with hypogammaglobulinemia and 1 with celiac disease. The patient with celiac disease recovered from autoimmune cytopenia after starting gluten-free diet. Cytopenias had continued in 14 of the patients, 11 of them had a least one recurrence. The Clinical Features of Patients are given in Table II. None of these patients had received bone marrow transplantation. No malignancy was detected. One patient with CVID died.

\section{Discussion}

Cytopenia is one of the most common autoimmune manifestations in immune disorders. The clinical course and significance of autoimmune cytopenias due to immunodeficiency or autoimmune diseases in children were evaluated in current study. Twenty-three of the patients with chronic or refractory autoimmune cytopenia $(6.8 \%)$ had an immunodeficiency or an autoimmune disease. Currently, there are a few reports examining the autoimmune cytopenias in childhood. In their large series with 326 CVID patients, Wang et al. $^{10}$ reported that $11 \%$ of patients had a history of autoimmune hematologic disease and 19 of patients (5.8\%) had the first episode of thrombocytopenia or AIHA prior to the diagnosis of immunodeficiency. A slight male predominance was observed. The ratio of male/female $(\mathrm{M} / \mathrm{F})$ was found 1.3. While this ratio has reported as $<1$ in adults, it has reported as variable in childhood $(\mathrm{M} / \mathrm{F}=0.7-$ 2.7) in previously studies. Also a tendency for male predominance in younger children was mentioned, similar to our finding. ${ }^{11,19}$

The median age of detection of cytopenia was 3.1 years (between 6 months-15.9 years). The median age at initial cytopenia was reported approximately median 5 years in children with chronic or refractory autoimmune cytopenia in previous studies (5-5.7 years). ${ }^{7,9,11} \mathrm{We}$ found that the diagnosis of primary disease was delayed up to 77 months (median 5 months) after cytopenias had occurred. There are currently a few reports examining the delay between the occurrence of cytopenia and diagnosis of immune disorders. Savaşan et al. ${ }^{20}$ reported that the delay of diagnosis in patients with CVID was approximately 8 years. Wang et al. ${ }^{10}$ reported that $5.8 \%$ of patients had the autoimmune cytopenia prior to the diagnosis of immunodeficiency and they reported that ITP occurs a median of 2 years before a diagnosis of CVID can be made. In a French Cohort, onset of Evans syndrome (ES) was concurrent with diagnosis of SLE in 3/13 patients, but was later within a 1 - to 10 year period in $10 / 13$ patients. ${ }^{11}$

A total of 13 patients (56.5\%) had singlelineage cytopenia and 10 patients (46.5\%) had multi-lineage cytopenia in our study. The most frequent single-lineage cytopenia was thrombocytopenia, followed by anemia, in accordance with reports in the literature. ${ }^{5,21-24}$ The percentage of multi-lineage cytopenia has been given different ratios in previous studies: Some of studies have given its ratio as $52-60.9 \%$ in their series. ${ }^{5,22-25}$ Also, Bride et al. ${ }^{7}$ reported that $24 / 30$ patients had multi-lineage cytopenia in patients with relapsed/refractory autoimmune cytopenia. However, Resnick et al. $^{23}$ have reported that $4.2 \%$ percentage of CVID patients have multi-lineage cytopenia.

Different definitions for ES have been made in the previous studies. Some of them defined it as a multi-lineage cytopenia due to autoimmune destruction of the cells, others defined it as AIHA and ITP with or without AIN. ${ }^{4,5,11,26-}$ 29 Recently, in a French National cohort study, they observed that ES is associated with immune manifestations including hypogammaglobulinemia. ${ }^{11}$ As a result, it is suggested that patients with ES (defined as an autoimmune multi-lineage cytopenias) should be screened for ALPS, CVID, and SLE. ${ }^{4}$

Three of the patients were diagnosed with CVID and 2 of the patients were diagnosed with ALPS. Approximately 20\% percentage of patients with CVID can be diagnosed at less than 20 years of age..$^{23}$ Also, it was reported that, ALPS did not have a high incidence in children 
with Evans syndrome. ${ }^{4}$ So, the scantiness of patients with CVID and ALPS may be due to the study being made with a pediatric population. Eight of the patients $(34.7 \%)$ were diagnosed with hypogammaglobulinemia (deficiency of IgA and/or IgG). Immune thrombocytopenia has been reported to occur in approximately $1 / 200$ of patients with selective IgA deficiency. Also, ITP and AIHA were seen in selective IgA deficiency which has been reported in previous studies. ${ }^{21,30}$ It is reported that various associated immune clinical or laboratory manifestations (lymphoproliferation, other autoimmune disease, and hypogammaglobulinemia) were observed in 93 children $(60 \%)$ with ES. ${ }^{11}$ As a possibility, maybe some of our patients with hypogammaglobulinemia will fulfill the criteria for ALPS, CVID or SLE in the future.

A total of 5 patients had beem diagnosed as SLE. None of them had neutropenia. Hematologic manifestations have been reported as a percentage of $34-38 \%$ in childhood SLE. ${ }^{26}$ Also, it was reported that, autoimmune cytopenias were significantly more frequent in the childhood SLE group. ${ }^{31}$ Hematologic abnormalities associated with SLE can include lymphopenia, hemolytic anemia and thrombocytopenia. However, neutropenia is uncommon in SLE. ${ }^{22-34}$ Our results are compatible with previous studies for SLE patients. Moreover, it is reported that the absence of neutropenia in children with SLE suggests that AIN is not a relevant mechanism in this disease. ${ }^{26}$

Two of the patients were with diagnosis of WAS. Thrombocytopenia is one of the major component of WAS. Pathophysiology of thrombocytopenia in patients with WAS is not clear but generally anomaly in actin cytoskeleton has been accused. ${ }^{35}$ Beside this, immune mediated clearance of platelets may have a role in thrombocytopenia of patients with WAS. ${ }^{36}$ Recently, it was shown that WAS is linked to defective regulatory $\mathrm{T}$ (Treg) cells and impaired $\mathrm{T}$ cell development. ${ }^{8}$ Therefore we included these patients in our study.
Five of the patients with multi-lineage cytopenias required second-line treatments. In a large series, Aladjidi et al. ${ }^{11}$ reported that $69 \%$ of their patients had needed several secondline treatments in children with ES. It is known that the management of patients with chronicrefractory autoimmune cytopenias is complex. Corticosteroids and IVIG are recommended as first-line therapy in a newly diagnosed patient. Second-line therapies are varied depending on the cell lineages affected and underlying disease. For example, patients with autoimmune cytopenias due to SLE are typically treated with medications for SLE, patients with ALPS are often treated with corticosteroids or MMF, whereas patients with CVID are often treated with IVIG per month. ${ }^{3,4,7,12}$

In the current study, none of the patient treated with rituximab had recovered from cytopenia. A number of studies have shown remarkable efficacy with rituximab in autoimmune cytopenias because it targets B cells. ${ }^{3,4,22}$ It is suggested that when a patient does not respond to rituximab, a calcineurin inhibitor may be used because the underlying autoimmune disease can be caused by a T-cell disorder. Also, it is reported that a patient with partial response to rituximab may benefit from a drug that suppresses both B and T cells, such as MMF. ${ }^{4}$ However, therapies for autoimmune cytopenias may still be insufficient since we still do not know much about the biology of autoimmune cytopenias. As a result, often times these patients are treated similarly to patients with primary disease. ${ }^{3,4,22}$ As we improve our understanding about the underlying biological mechanisms, new treatment options including bortezomib, belimumab, tocilizumab and epratuzumab will be used for autoimmune cytopenias. ${ }^{11}$ Recently, sirolimus has been recommended as a first or second line agent for patients with chronicrefractory autoimmune cytopenias 2016. During our study, none of our patients were treated with those agents.

Two patients who failed to respond to medical treatment had to undergo splenectomy. Both of 
them had hypogammaglobulinemia. If a patient has an immune deficiency, splenectomy should have been avoided. However, sometimes splenectomy may be opted for patients who have persistent diseases. ${ }^{4,12,27}$

In the current study, a total of 8 patients (34.7\%) recovered from cytopenia. The rate of recovery from autoimmune cytopenia in this study is similar to that in previous childhood studies. ${ }^{6,26}$ Cytopenias had continued in 14 patients and 11 of them had a least one recurrence. Recurrent episodes were treated in a similar manner. In a long-term outcome of a French study, 74\% of those patients (67/90) had experienced a relapse. ${ }^{11}$

No malignancy was detected in our patients. Various malignancies (including lymphoma, Hodgkin's disease, or breast cancer) have been reported in patients with immunodeficiency or autoimmune disease. ${ }^{11}$ However, it has been reported that none were diagnosed with malignancy. ${ }^{11}$

One patient with CVID died. No patient death has been reported for autoimmune cytopenias due to SLE, whereas 7-36\% mortality rates have been reported for autoimmune cytopenias due to other immune disorders. ${ }^{11,26}$

Cytopenia may be the first finding of an immunodeficiency or autoimmune disease and primary disease may be diagnosed during the clinical course. Taking the new targeted treatment options into consideration; early diagnosis is likely to become more important in the near-future in order to begin treatment for the underlying disease as early as possible.

\section{REFERENCES}

1. Miano M, Scalzone M, Perri K, et al. Mycophenolate mofetil and Sirolimus as second or further line treatment in children with chronic refractory Primitive or Secondary Autoimmune Cytopenias: a single centre experience. Br J Haematol 2015; 171: 247-253.
2. Price V. Auto-immune lymphoproliferative disorder and other secondary immune thrombocytopenias in childhood. Pediatr Blood Cancer 2013; 60(Suppl 1): S12-S14.

3. Seidel MG. Autoimmune and other cytopenias in primary immunodeficiencies: pathomechanisms, novel differential diagnoses, and treatment. Blood 2014; 124: 2337-2344.

4. Teachey DT, Lambert MP. Diagnosis and management of autoimmune cytopenias in childhood. Pediatr Clin North Am 2013; 60: 14891511.

5. Al Ghaithi I, Wright NA, Breakey VR, et al. Combined autoimmune cytopenias presenting in childhood. Pediatr Blood Cancer 2016; 63: 292-298.

6. Aladjidi N, Leverger G, Leblanc T, et al; Centre de Référence National des Cytopénies Auto-immunes de l'Enfant (CEREVANCE). New insights into childhood autoimmune hemolytic anemia: a French National Observational Study of 265 children. Haematologica 2011; 96: 655-663.

7. Bride KL, Vincent $\mathrm{T}$, Smith-Whitley $\mathrm{K}$, et al. Sirolimus is effective in relapsed/refractory autoimmune cytopenias: results of a prospective multi-institutional trial. Blood 2016; 127: 17-28.

8. Ghosh S, Seidel MG. Editorial: Current challenges in immune and other acquired cytopenias of childhood. Front Pediatr 2016; 4: 3.

9. Rao VK. Approaches to managing autoimmune cytopenias in novel immunological disorders with genetic underpinnings like autoimmune lymphoproliferative syndrome. Front Pediatr 2015; 3: 65 .

10. Wang J, Cunningham-Rundles C. Treatment and outcome of autoimmune hematologic disease in common variable immunodeficiency (CVID). J Autoimmun 2005; 25: 57-62.

11. Aladjidi N, Fernandes H, Leblanc T, et al. Evans syndrome in children: long-term outcome in a Prospective French National Observational Cohort. Front Pediatr 2015; 3: 79.

12. Neunert C, Lim W, Crowther M, Cohen A, Solberg L Jr, Crowther MA; American Society of Hematology. The American Society of Hematology 2011 evidence-based practice guideline for immune thrombocytopenia. Blood 2011; 117: 4190-4207.

13. Kliegman RM, Behrman RE, Jenson HB, Stanton BF. Nelson Textbook of Pediatrics. (18th ed). Philadelphia: Saunders Elsevier, 2007.

14. Dale DC, Bolyard AA, Schwinzer BG, et al. The severe chronic neutropenia International Registry: 10-year follow-up report. Support Cancer Ther 2006; 3: 220-231. 
15. Chapel H, Cunningham-Rundles C. Update in understanding common variable immunodeficiency disorders (CVIDs) and the management of patients with these conditions. Br J Haematol 2009; 145: 709727.

16. Bousfiha AA, Jeddane L, Ailal F, et al. A phenotypic approach for IUIS PID classification and diagnosis: guidelines for clinicians at the bedside. J Clin Immunol 2013; 33: 1078-1087.

17. Oliveira JB, Bleesing JJ, Dianzani U, et al. Revised diagnostic criteria and classification for the autoimmune lymphoproliferative syndrome (ALPS): report from the 2009 NIH International Workshop. Blood 2010; 116: e35-e40.

18. Petri M, Orbai AM, Alarcón GS, et al. Derivation and validation of the Systemic Lupus International Collaborating Clinics classification criteria for systemic lupus erythematosus. Arthritis Rheum 2012; 64: 2677-2686.

19. Sipurzynski J, Fahrner B, Kerbl R, et al. Management of chronic immune thrombocytopenia in children and adolescents: lessons from an Austrian national cross-sectional study of 81 patients. Semin Hematol 2016; 53(Suppl 1): S43-S47.

20. Savaşan S, Warrier I, Buck S, Kaplan J, Ravindranath Y. Increased lymphocyte Fas expression and high incidence of common variable immunodeficiency disorder in childhood Evans' syndrome. Clin Immunol (Orlando, Fla) 2007; 125: 224-229.

21. Cunningham-Rundles $C$. Hematologic complications of primary immune deficiencies. Blood Rev 2002; 16: 61-64.

22. Cunningham-Rundles C. How I treat common variable immune deficiency. Blood 2010; 116: 7-15.

23. Resnick ES, Moshier El, Godbold JH, CunninghamRundles C. Morbidity and mortality in common variable immune deficiency over 4 decades. Blood 2012; 119: 1650-1657.

24. Warnatz K, Voll RE. Pathogenesis of autoimmunity in common variable immunodeficiency. Front Immunol 2012; 3: 210.

25. Neven B, Magerus-Chatinet A, Florkin B, et al. A survey of 90 patients with autoimmune lymphoproliferative syndrome related to TNFRSF6 mutation. Blood 2011; 118: 4798-4807.
26. Lube GE, Ferriani MPL, Campos LMA, et al. Evans syndrome at childhood-onset systemic lupus erythematosus diagnosis: a large multicenter study. Pediatr Blood Cancer 2016; 63: 1238-1243.

27. Podjasek JC, Abraham RS. Autoimmune cytopenias in common variable immunodeficiency. Front Immunol 2012; 3: 189.

28. Sankaran J, Rodriguez V, Jacob EK, Kreuter JD, Go RS. Autoimmune hemolytic anemia in children: Mayo Clinic Experience. J Pediatr Hematol Oncol 2016; 38: e120-e124.

29. Wang WC. Evans syndrome in childhood: pathophysiology, clinical course, and treatment. Am J Pediatr Hematol Oncol 1988; 10: 330-338.

30. Hammarström L, Vorechovsky I, Webster D. Selective IgA deficiency (SIgAD) and common variable immunodeficiency (CVID). Clin Exp Immunol 2000; 120: 225-231.

31. Livingston B, Bonner A, Pope J. Differences in clinical manifestations between childhood-onset lupus and adult-onset lupus: a meta-analysis. Lupus 2011; 20: 1345-1355.

32. Miescher PA, Tucci A, Beris P, Favre H. Autoimmune hemolytic anemia and/or thrombocytopenia associated with lupus parameters. Semin Hematol 1992; 29: 13-17.

33. Iqbal S, Sher MR, Good RA, Cawkwell GD. Diversity in presenting manifestations of systemic lupus erythematosus in children. J Pediatr 1999; 135: 500505.

34. Schmugge M, Revel-Vilk S, Hiraki L, Rand ML, Blanchette VS, Silverman ED. Thrombocytopenia and thromboembolism in pediatric systemic lupus erythematosus. J Pediatr 2003; 143: 666-669.

35. Shcherbina A, Rosen FS, Remold-O'Donnell E. Pathological events in platelets of Wiskott-Aldrich syndrome patients. Br J Haematol 1999; 10: 875-883.

36. Marathe BM, Prislovsky A, Astrakhan A, Rawlings DJ, Wan JY, Strom TS. Antiplatelet antibodies in WASP(-) mice correlate with evidence of increased in vivo platelet consumption. Exp Hematol 2009; 37: 1353-1363. 